\title{
Iterated Function Systems Consisting of Generalized Convex Contractions in the Framework of Complete Strong b-metric Spaces
}

Flavian Georgescu

\begin{abstract}
The concept of generalized convex contraction was introduced and studied by V. Istrăţescu and the notion of $b$-metric space was introduced by I. A. Bakhtin and S. Czerwik. In this paper we combine these two elements by studying iterated function systems consisting of generalized convex contractions on the framework of $b$-metric spaces. More precisely we prove the existence and uniqueness of the attractor of such a system providing in this way a generalization of Istrăţescu's convex contractions fixed point theorem in the setting of complete strong $b$-metric spaces.
\end{abstract}

AMS Subject Classification (2010). 28A80; 54H20

Keywords. iterated function systems consisting of generalized convex contractions, $b$-metric spaces, fixed points

\section{Introduction}

Iterated function systems, whose foundations are due to J. Hutchinson (see $[26]$ ), represent one of the basic ways to construct fractals.

Two directions of generalization of this concept are the following: 
- to consider more general domains or ranges of the iterated function systems (see, for example, [6], [10], [13], [14], [24], [30], [33] and [45]).

- to work with more general contractive conditions on the constitutive functions of the iterated function systems (see, for example, [18], [34], [36], [37], [38], [39], [45], [48], [52], [53], [57] and [58]).

Concerning the first line of generalization, we emphasize the papers [10], [14] and [45], were iterated function systems in the setting of $b$-metric spaces are studied. The notion of $b$-metric space was introduced by I. A. Bakhtin (see [8]) and S. Czerwik (see [15] and [16]). In the last years a lot of fixed point results in the framework of $b$-metric spaces have been obtained (see, for example, [1], [7], [9], [11], [12], [17], [27], [28], [29], [31], [40], [41], [42], [44], [46], [47], [49], [50], [51], [55], [56] and [59]).

Concerning the second line of generalization, of special interest for the present study, is the paper [39] where the notion of iterated function system consisting of convex contractions is introduced and the existence and uniqueness of the attractor of such a system were obtained. The concept of generalized convex contraction was introduced by V. Istrățescu and studied also by S. András (see [4], [5], [19], [20] and [21]):

Definition 1.1. Given a complete metric space $(X, d)$, a continuos function $f: X \rightarrow X$ is called a generalized convex contraction provided that there exist $m \in \mathbb{N}^{*}$ and $\alpha_{0}, \alpha_{1}, \ldots, \alpha_{m-1} \geq 0$ such that $\sum_{i=0}^{m-1} \alpha_{i}<1$ and $d\left(f^{[m]}(x), f^{[m]}(y)\right) \leq \sum_{k=0}^{m-1} \alpha_{k} d\left(f^{[k]}(x), f^{[k]}(y)\right)$ for all $x, y \in X$, where by $f^{[k]}$ we mean the composition of $f$ by itself $k$ times.

They proved that each continuous generalized convex contraction is a Picard operator. We note that for $m=2$ one obtains the concept of convex contraction. For other generalizations of Istrăţescu's result see [2], [23], [25], [32], [35] and [43].

The result presented in this paper fits into both directions of generalizations presented above. More precisely, we study iterated function systems consisting of generalized convex contractions (illustrating the second direction) on the framework of complete (strong) $b$-metric spaces (illustrating the first direction).

On the one hand, it is our duty to underline the strong influence of the paper [39] on our work, the main lines of arguments used in this article being adaptations of the ones used there to the framework of $b$-metric spaces. The proofs of Propositions 3.6 and 3.7 are unchanged with respect to those from 
the aforementioned paper, but we included them for the sake of completeness of the presentation.

On the other hand, we should emphasize that there are some big differences between the setting of metric spaces and the one of $b$-metric spaces which give raise to some problems in certain approaches. Given a $b$-metric space $(X, d, s), x \in X, r>0,\left(x_{n}\right)_{n \in \mathbb{N}}$ and $\left(y_{n}\right)_{n \in \mathbb{N}}$ sequences of elements from $X$ and $u, v \in X$ such that $\lim _{n \rightarrow \infty} x_{n}=u$ and $\lim _{n \rightarrow \infty} y_{n}=v$, we mention some of these differences:

$-\{y \in X \mid d(x, y)<r\}$ is not necessary open (see [3]);

- $\{y \in X \mid d(x, y) \leq r\}$ is not necessary closed;

$-d$ is not necessary continuous (actually we have

$$
\frac{1}{s^{2}} d(u, v) \leq \varliminf_{n \rightarrow \infty} d\left(x_{n}, y_{n}\right) \leq \varlimsup_{n \rightarrow \infty} d\left(x_{n}, y_{n}\right) \leq s^{2} d(u, v)
$$

and

$$
\frac{1}{s} d(u, x) \leq \varliminf_{n \rightarrow \infty} d\left(x_{n}, x\right) \leq \varlimsup_{n \rightarrow \infty} d\left(x_{n}, x\right) \leq s d(u, x)
$$

see [42], [44] and [49]).

\section{Preliminaries}

\section{Notations concerning functions and sets}

In the sequel, $f^{[n]}$ designates the composition of the function $f: X \rightarrow X$ by itself $n$ times.

For a family of functions $\left(f_{i}\right)_{i \in I}$, where $f_{i}: X \rightarrow X, \alpha_{1}, \alpha_{2}, \ldots, \alpha_{n} \in I$ and $Y \subseteq X$, we use the following notations:

$$
\begin{gathered}
f_{\alpha_{1}} \circ f_{\alpha_{2}} \circ \ldots \circ f_{\alpha_{n}} \stackrel{\text { not }}{=} f_{\alpha_{1} \alpha_{2} \ldots \alpha_{n}} \\
f_{\alpha_{1} \alpha_{2} \ldots \alpha_{n}}(Y) \stackrel{\text { not }}{=} Y_{\alpha_{1} \alpha_{2} \ldots \alpha_{n}} .
\end{gathered}
$$

$B^{A}$ means the set of functions from $A$ to $B$.

For a set $I$, we use the following notations:

$$
I^{\mathbb{N}^{*}} \stackrel{\text { not }}{=} \Lambda(I)
$$

so the elements of $\Lambda(I)$ can be presented as infinite words $\omega=\omega_{1} \omega_{2} \ldots \omega_{n} \ldots$ with letters from $I$

$$
I^{\{1,2, \ldots, m\}} \stackrel{\text { not }}{=} \Lambda_{m}(I)
$$


hence the elements of $\Lambda(I)$ are words $\omega=\omega_{1} \omega_{2} \ldots \omega_{m}$ with $m$ letters from $I$

$$
\Lambda_{0}(I) \cup \Lambda_{1}(I) \cup \ldots \cup \Lambda_{m-1}(I) \stackrel{\text { not }}{=} V_{m}(I),
$$

where $\Lambda_{0}(I)=\{\lambda\}$ is the set consisting on the empty word; hence $V_{m}(I)$ is the set of all words having at most $m-1$ letters from $I$

$$
\bigcup_{n \in \mathbb{N}} \Lambda_{n}(I) \stackrel{\text { not }}{=} \Lambda^{*}(I)
$$

thus $\Lambda^{*}(I)$ is the set of all finite words with letters from $I$.

For a function $f: X \rightarrow X$, by $f_{\lambda}$ we mean $I d_{X}: X \rightarrow X$ given by

$$
\operatorname{Id}_{X}(x)=x
$$

for every $x \in X$.

For $\alpha \in \Lambda(I) \cup \Lambda_{n}(I)$ and $m, n \in \mathbb{N}, m \leq n$, we use the following notation:

$$
\alpha_{1} \alpha_{2} \ldots \alpha_{m} \stackrel{\text { not }}{=}[\alpha]_{m}
$$

Given the words $\alpha \in \Lambda^{*}(I)$ and $\beta \in \Lambda(I) \cup \Lambda^{*}(I)$, by $\alpha \beta$ we understand the concatenation of them.

\section{Basic facts concerning $b$-metric spaces}

The original impulse of the study of $b$-metric spaces comes from measure theory.

Definition 2.1. Given a nonempty set $X$ and a real number $s \in[1, \infty)$, a function $d: X \times X \rightarrow[0, \infty)$ is called b-metric if it satisfies the following properties:

i) $d(x, y)=0$ if and only if $x=y$;

ii) $d(x, y)=d(y, x)$ for all $x, y \in X$;

iii) $d(x, y) \leq s(d(x, z)+d((z, y))$ for all $x, y, z \in X$.

We denote such a system by $(X, d, s)$ and we call it a $b$-metric space with constant $s$.

The classic examples of $b$-metric spaces are $l^{p}(\mathbb{R})$ and $L^{p}[0,1]$, where $p \in$ $(0,1)$. Some other examples of $b$-metric spaces could be found in [7], [9], [12], [15] and [16].

Remark 2.1. Every metric space is a $b$-metric space with constant 1 , but there exist $b$-metric spaces which are not metric spaces. 
Indeed, let $X=\{0,1,2\}$ and set

$$
\begin{gathered}
d(0,0)=d(1,1)=d(2,2)=0, \\
d(0,1)=d(1,0)=d(0,2)=d(2,0)=1
\end{gathered}
$$

and

$$
d(1,2)=d(2,1)=3 .
$$

Then:

$-(X, d)$ is a $b$-metric space since

$$
d(x, y) \leq \frac{3}{2}(d(x, z)+d(z, y))
$$

for all $x, y, z \in X$

$-(X, d)$ is not a metric space since

$$
d(1,2)=3>d(1,0)+d(0,2)=2 .
$$

For similar examples see [12], [15] and [16].

Lemma 2.1. (see [15] and [16]). For every b-metric space $(X, d, s), p \in \mathbb{N}$ and $x_{0}, x_{1}, \ldots, x_{p} \in X$ we have

$$
d\left(x_{0}, x_{p}\right) \leq \sum_{i=1}^{p} s^{i} d\left(x_{i-1}, x_{i}\right)
$$

Definition 2.2. A sequence $\left(x_{n}\right)_{n \in \mathbb{N}}$ of elements from a b-metric space $(X, d, s)$ is called:

- convergent if there exists $l \in \mathbb{R}$ such that

$$
\lim _{n \rightarrow \infty} d\left(x_{n}, l\right)=0
$$

- Cauchy if $\lim _{m, n \rightarrow \infty} d\left(x_{m}, x_{n}\right)=0$, i.e. for every $\varepsilon>0$ there exists $n_{\varepsilon} \in \mathbb{N}$ such that

$$
d\left(x_{m}, x_{n}\right)<\varepsilon
$$

for all $m, n \in \mathbb{N}, m, n \geq n_{\varepsilon}$.

The b-metric space $(X, d, s)$ is called complete if every Cauchy sequence of elements from $X$ is convergent.

The next result turns out to be a crucial tool in the proof of Propositions 3.1 and 3.2. 
Lemma 2.2. (see Corollary 2.8 from [40]). Let $\left(x_{n}\right)_{n \in \mathbb{N}}$ be a sequence of elements from a $b$-metric space $(X, d, s)$. If there exists $\alpha>1$ such that the series $\sum_{n=1}^{\infty} \alpha^{n} d\left(x_{n}, x_{n+1}\right)$ is convergent, then the sequence $\left(x_{n}\right)_{n \in \mathbb{N}}$ is Cauchy.

Remark 2.2. We endow a $b$-metric space with the topology induced by its convergence.

In particular:

- the closure $\bar{Y}$ of a subset of a $b$-metric space $(X, d, s)$ is defined in the following way:

$$
\begin{gathered}
\bar{Y}=\left\{x \in X \mid \text { there exists }\left(x_{n}\right)_{n \in \mathbb{N}} \text { a sequence of elements from } Y\right. \\
\text { such that } \left.\lim _{n \rightarrow \infty} x_{n}=x\right\} ;
\end{gathered}
$$

- a function $f: A \rightarrow X$, where $A$ is a subset of a $b$-metric space $(X, d, s)$, is continuous if $\lim _{n \rightarrow \infty} f\left(x_{n}\right)=f(l)$ for every sequence $\left(x_{n}\right)_{n \in \mathbb{N}}$ of elements from $A$ converging to $l \in A$.

Since, as we have mentioned in the Introduction, in an arbitrary $b$-metric space, the distance $d$ is not necessary continuous, we introduce a strengthening of the notion of $b$-metric space which eludes this drawback.

Definition 2.3. Given a nonempty set $X$ and a real number $s \in[1, \infty)$, a function $d: X \times X \rightarrow[0, \infty)$ is called a strong b-metric if it satisfies the following properties:

i) $d(x, y)=0$ if and only if $x=y$;

ii) $d(x, y)=d(y, x)$ for all $x, y \in X$;

iii) $d(x, y) \leq d(x, z)+s d(z, y)$ for all $x, y, z \in X$.

By abuse of notation, we denote such a system by $(X, d, s)$ and we call it a strong $b$-metric space with constant $s$.

Remark 2.3. Every metric space is a strong 1 -metric space. Every strong $b$-metric space with constant $s$ is a $b$-metric space with constant $s$.

Proposition 2.3. (see pages 122 and 123 from $[29])$. Let $(X, d, s)$ be a strong $b$-metric space. Then $\lim _{n \rightarrow \infty} d\left(x_{n}, y_{n}\right)=d(x, y)$ for every $\left(x_{n}\right)_{n \in \mathbb{N}},\left(y_{n}\right)_{n \in \mathbb{N}}$ sequences of elements of $X$ and $x, y \in X$ such that $\lim _{n \rightarrow \infty} x_{n}=x$ and $\lim _{n \rightarrow \infty} y_{n}=$ $y$, i.e. $d$ is continuous. 


\section{Basic facts concerning the Hausdorff-Pompeiu generalized metric in the setting of $b$-metric spaces}

Following the notations from [10], we shall deal with the following families of subsets of a $b$-metric space $(X, d, s)$ :

$\infty\}$

$-\mathcal{P}(X)=\{Y \mid Y \subseteq X\}$

- $P(X)=\{Y \in \mathcal{P}(X) \mid Y \neq \emptyset\}$

- $P_{b}(X)=\{Y \in P(X) \mid Y$ is bounded $\}=\left\{Y \in P(X) \mid \sup _{x, y \in Y} d(x, y)<\right.$

- $P_{c l}(X)=\{Y \in P(X) \mid Y$ is closed $\}$, where $Y$ closed means that for every sequence of elements of $Y$ converging to $x \in X$ we have $x \in Y$

- $P_{b, c l}(X)=P_{b}(X) \cap P_{c l}(X)$

- $P_{c p}(X)=\{Y \in P(X) \mid Y$ is compact $\}$, where $Y$ compact means that for every sequence of elements of $Y$ there exists a subsequence converging to an element of $Y$.

Note that one can easily check, using the technique used in the case of the metric spaces with very minor changes, that:

$$
P_{c p}(X) \subseteq P_{b, c l}(X) .
$$

Definition 2.4. For a $b$-metric space $(X, d, s)$, we consider the HausdorffPompeiu generalized metric $H: \mathcal{P}(X) \times \mathcal{P}(X) \rightarrow[0,+\infty]$ described by

$$
\begin{gathered}
H(A, B)=\max \left\{\sup _{x \in A}\left(\inf _{y \in B} d(x, y)\right), \sup _{x \in B}\left(\inf _{y \in A} d(x, y)\right)\right\}= \\
=\inf \left\{\delta \in[0, \infty] \mid A \subseteq E_{\delta}(B) \text { and } B \subseteq E_{\delta}(A)\right\},
\end{gathered}
$$

for every $A, B \in \mathcal{P}(X)$, where $E_{\delta}(A)$ is the $\delta$-expansion of $A$, i.e.

$$
E_{\delta}(A)=\bigcup_{x \in A} B(x, \delta)=\{y \in X \mid \text { there exists } x \in A \text { such that } d(x, y)<\delta\} .
$$

Proposition 2.4. i) For a strong $b$ - metric space $(X, d, s)$, we have:

$$
H(A, B)=H(\bar{A}, \bar{B}),
$$

for every $A, B \in \mathcal{P}(X)$;

ii) For a $b$ - metric space $(X, d, s)$, we have:

$$
H(A \cup B, C \cup D) \leq \max \{H(A, C), H(B, D)\},
$$


for every $A, B, C, D \in \mathcal{P}(X)$. Moreover, we have

$$
H\left(\bigcup_{i \in I} A_{i}, \bigcup_{i \in I} B_{i}\right) \leq \sup _{i \in I} H\left(A_{i}, B_{i}\right),
$$

for every families $\left(A_{i}\right)_{i \in I},\left(B_{i}\right)_{i \in I}$ of elements from $\mathcal{P}(X)$.

Proof.

i) Let us consider arbitrary, but fixed, $A, B \in \mathcal{P}(X)$.

We claim that

$$
\sup _{x \in A}\left(\inf _{y \in B} d(x, y)\right)=\sup _{x \in \bar{A}}\left(\inf _{y \in \bar{B}} d(x, y)\right) .
$$

Indeed, it is clear that, for a fixed $x \in X$, we have $\inf _{y \in \bar{B}} d(x, y) \leq \inf _{y \in B} d(x, y)$. If, by reductio ad absurdum, $\inf _{y \in \bar{B}} d(x, y)<\inf _{y \in B} d(x, y)$, then there exists $c \in$ $\mathbb{R}$ such that $\inf _{y \in \bar{B}} d(x, y)<c<\inf _{y \in B} d(x, y)$ and, consequently, one can find $y_{0} \in \bar{B}$ such that $d\left(x, y_{0}\right)<c$. Thus, there exists a sequence $\left(u_{n}\right)_{n \in \mathbb{N}}$ of elements from $B$ having the property that $\lim _{n \rightarrow \infty} u_{n}=y_{0}$. Since $d\left(x, y_{0}\right)<$ $c<\inf _{y \in B} d(x, y) \leq d\left(x, u_{n}\right)$ for all $n \in \mathbb{N}$, by passing to limit as $n$ goes to $\infty$ in the previous inequality and taking into account Proposition 2.3, we get the contradiction $d\left(x, y_{0}\right)<d\left(x, y_{0}\right)$. Therefore $\inf _{y \in \bar{B}} d(x, y)=\inf _{y \in B} d(x, y)$ for every $x \in A$. Consequently $\sup _{x \in A}\left(\inf _{y \in B} d(x, y)\right) \leq \sup _{x \in \bar{A}}\left(\inf _{y \in \bar{B}} d(x, y)\right)$. If, by reductio ad absurdum, $\sup _{x \in A}\left(\inf _{y \in B} d(x, y)\right)<\sup _{x \in \bar{A}}\left(\inf _{y \in \bar{B}} d(x, y)\right)$, there is $c \in \mathbb{R}$ such that $\sup _{x \in A}\left(\inf _{y \in B} d(x, y)\right)<c<\sup _{x \in \bar{A}}\left(\inf _{y \in \bar{B}} d(x, y)\right)$ and, consequently, one can find $x_{0} \in \bar{A}$ such that $c<\inf _{y \in \bar{B}} d\left(x_{0}, y\right)$. There exists a sequence $\left(v_{n}\right)_{n \in \mathbb{N}}$ of elements from $A$ having the property that $\lim _{n \rightarrow \infty} v_{n}=x_{0}$. Since, for an arbitrary, but fixed, $n \in \mathbb{N}$, we have $d\left(x_{0}, y\right) \stackrel{n \rightarrow \infty}{\leq} d\left(v_{n}, y\right)+s d\left(v_{n}, x_{0}\right)$ for every $y \in \bar{B}$, we get $\inf _{y \in \bar{B}} d\left(x_{0}, y\right)-\inf _{y \in \bar{B}} d\left(v_{n}, y\right) \leq s d\left(v_{n}, x_{0}\right)$. Interchanging the roles of $x_{0}$ and $v_{n}$, one obtains $\inf _{y \in \bar{B}} d\left(v_{n}, y\right)-\inf _{y \in \bar{B}} d\left(x_{0}, y\right) \leq s d\left(v_{n}, x_{0}\right)$. Consequenly $\left|\inf _{y \in \bar{B}} d\left(x_{0}, y\right)-\inf _{y \in \bar{B}} d\left(v_{n}, y\right)\right| \leq s d\left(v_{n}, x_{0}\right)$ for all $n \in \mathbb{N}$ and since $\lim _{n \rightarrow \infty} d\left(v_{n}, x_{0}\right)=0$, we get

$$
\lim _{n \rightarrow \infty} \inf _{y \in \bar{B}} d\left(v_{n}, y\right)=\inf _{y \in \bar{B}} d\left(x_{0}, y\right)
$$


Therefore, since

$$
\inf _{y \in \bar{B}} d\left(v_{n}, y\right)=\inf _{y \in B} d\left(v_{n}, y\right) \leq \sup _{x \in A}\left(\inf _{y \in B} d(x, y)\right)<c<\inf _{y \in \bar{B}} d\left(x_{0}, y\right)
$$

for all $n \in \mathbb{N}$, by passing to limit as $n$ goes to $\infty$ in the previous inequality and taking into account (2), we get the contradiction $\inf _{y \in \bar{B}} d\left(x_{0}, y\right) \leq c<$ $\inf _{y \in \bar{B}} d\left(x_{0}, y\right)$. Thus $\sup _{x \in A}\left(\inf _{y \in B} d(x, y)\right)=\sup _{x \in \bar{A}}\left(\inf _{y \in \bar{B}} d(x, y)\right)$.

In a similar way one can prove that

$$
\sup _{x \in B}\left(\inf _{y \in A} d(x, y)\right)=\sup _{x \in \bar{B}}\left(\inf _{y \in \bar{A}} d(x, y)\right) .
$$

From (1) and (3) we conclude that $H(A, B)=H(\bar{A}, \bar{B})$.

ii) The proof runs like the one for the classic framework of metric spaces (see Theorem 1.13, page 22, from [54]). For the first part of ii) one can also consult [45].

Proposition 2.5. (see [16]) If the b-metric space $(X, d, s)$ is complete, then $\left(P_{c p}(X), H, s\right)$ and $\left(P_{c l}(X), H, s\right)$ are complete $b$ - metric spaces.

Moreover,

$$
H(A, B) \leq s(H(A, C)+H(C, B))
$$

for every $A, B, C \in \mathcal{P}(X)$.

Definition 2.5. For a b-metric space $(X, d, s)$, we consider the function $\delta: P_{c p}(X) \times P_{c p}(X) \rightarrow[0,+\infty)$ defined by

$$
\delta\left(K_{1}, K_{2}\right)=\sup _{x \in K_{1}, y \in K_{2}} d(x, y)
$$

for all $K_{1}, K_{2} \in P_{c p}(X)$.

In particular, for every $K \in P_{c p}(X)$, we consider

$$
\operatorname{diam}(K) \stackrel{\text { def }}{=} \delta(K, K)=\sup _{x, y \in K} d(x, y) .
$$

One can easily check the following:

Remark 2.4. For a $b$-metric space $(X, d, s)$, we have

$$
H\left(K_{1}, K_{2}\right) \leq \delta\left(K_{1}, K_{2}\right)
$$

for all $K_{1}, K_{2} \in P_{c p}(X)$. 
Justification. Let us consider $K_{1}, K_{2} \in P_{c p}(X)$ arbitrarily chosen, but fixed. For all $x \in K_{1}$ and $y \in K_{2}$, we have $\inf _{y \in K_{2}} d(x, y) \leq d(x, y) \leq$ $\sup _{x \in K_{1}, y \in K_{2}} d(x, y)=\delta\left(K_{1}, K_{2}\right)$, so $\sup _{x \in K_{1}} \inf _{y \in K_{2}} d(x, y) \leq \delta\left(K_{1}, K_{2}\right)$. In a similar way one can prove that $\sup _{x \in K_{2}} \inf _{y \in K_{1}} d(x, y) \leq \delta\left(K_{1}, K_{2}\right)$. Consequently $\max \left\{\sup _{x \in K_{1}} \inf _{y \in K_{2}} d(x, y), \sup _{x \in K_{2}} \inf _{y \in K_{1}} d(x, y)\right\} \leq \delta\left(K_{1}, K_{2}\right)$, i.e. $H\left(K_{1}, K_{2}\right) \leq \delta\left(K_{1}, K_{2}\right)$.

Proposition 2.6. Let $(X, d, s)$ be a complete strong $b$-metric space, $\left(K_{n}\right)_{n \in \mathbb{N}} \subseteq P_{c p}(X)$ and $K \in P_{c p}(X)$ such that $\lim _{n \rightarrow \infty} H\left(K_{n}, K\right)=0$. Then

$$
L \stackrel{\text { def }}{=} K \cup\left(\bigcup_{n=0}^{\infty} K_{n}\right) \in P_{c p}(X) .
$$

The proof of the above proposition runs in a similar way to that of Proposition 2.8 from [39] adapting the classic techniques from the metric spaces framework.

\section{The concept of Picard operator}

Definition 2.6. A function $f: X \rightarrow X$, where $(X, d, s)$ is a $b$-metric space, is called Picard operator if there exists a unique $x^{*} \in X$ such that $f\left(x^{*}\right)=x^{*}$ and $\lim _{k \rightarrow \infty} f^{[k]}(x)=x^{*}$ for every $x \in X$.

\section{The main result}

Definition 3.1. Given a natural number $m$, an iterated function system consisting of generalized convex contractions on a complete $b$ - metric space $(X, d, s)$ consists of a finite family of continuous functions $\left(f_{i}\right)_{i \in I}$, where $f_{i}: X \rightarrow X$, having the following property: for every $\omega \in \Lambda_{m}(I)$ there exists a family of positive numbers $\left(a_{\omega, v}\right)_{v \in V_{m}(I)}$ such that:

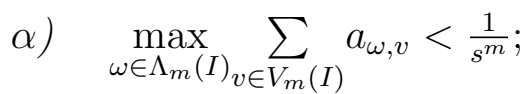

B) $d\left(f_{\omega}(x), f_{\omega}(y)\right) \leq \sum_{v \in V_{m}(I)} a_{\omega, v} d\left(f_{v}(x), f_{v}(y)\right)$, for all $\omega \in \Lambda_{m}(I), x, y \in X$.

Such a system will be denoted by

$$
\mathcal{S}=\left((X, d, s), m,\left(f_{i}\right)_{i \in I}\right)
$$


One can associate to such a system the function $F_{\mathcal{S}}: P_{c p}(X) \rightarrow P_{c p}(X)$ given by

$$
F_{\mathcal{S}}(K)=\bigcup_{i \in I} f_{i}(K)
$$

for every $K \in P_{c p}(X)$.

A fixed point of $F_{\mathcal{S}}$ is called attractor of $\mathcal{S}$.

In the sequel, for an iterated function system consisting of generalized convex contractions on a complete $b$-metric space $\mathcal{S}=\left((X, d, s), m,\left(f_{i}\right)_{i \in I}\right)$ we use the following notations:

$-\sum_{v \in V_{m}(I)} a_{\omega, v} \stackrel{\text { not }}{=} A_{\omega}$, where $\omega \in \Lambda_{m}(I)$

$$
-\max _{\omega \in \Lambda_{m}(I)} A_{\omega} \stackrel{\text { not }}{=} A<\frac{1}{s^{m}}
$$

$-A \cdot s^{m} \stackrel{\text { not }}{=} C<1$

$-x_{n}\left(K_{1}, K_{2}\right) \stackrel{\text { not }}{=} \max \left\{\delta\left(f_{\omega}\left(K_{1}\right), f_{\omega}\left(K_{2}\right)\right) \mid \omega \in \Lambda_{n}(I)\right\}$, where

$K_{1}, K_{2} \in P_{c p}(X), n \in \mathbb{N}^{*}$

$-y_{n}\left(K_{1}, K_{2}\right)=\max \left\{x_{n}\left(K_{1}, K_{2}\right), \ldots, x_{n-m+2}\left(K_{1}, K_{2}\right), x_{n-m+1}\left(K_{1}, K_{2}\right)\right\}$, where $n \in \mathbb{N}^{*}, n \geq m-1$ and $K_{1}, K_{2} \in P_{c p}(X)$.

When there is no danger of confusion, for the sake of simplicity, we shall use the following notations:

$$
y_{n}\left(K_{1}, K_{2}\right) \stackrel{\text { not }}{=} y_{n} \text { and } x_{n}\left(K_{1}, K_{2}\right) \stackrel{\text { not }}{=} x_{n} .
$$

Proposition 3.1. For every iterated function system consisting of generalzed convex contractions on a complete b-metric space $\mathcal{S}=\left((X, d, s), m,\left(f_{i}\right)_{i \in I}\right)$ there exists $A_{\mathcal{S}} \in P_{c p}(X)$ such that the sequence $\left(F_{\mathcal{S}}^{[n]}(K)\right)_{n \in \mathbb{N}^{*}}$ converges in the Hausdorff-Pompeiu metric to $A_{\mathcal{s}}$ for every $K \in P_{c p}(X)$.

Proof. Our proof is divided into four steps.

THE FIRST STEP is to show that the sequence $\left(y_{k+m}\left(K_{1}, K_{2}\right)\right)_{k \in \mathbb{N}}$ is decreasing for all $K_{1}, K_{2} \in P_{c p}(X)$.

Justification of the first step. Let us consider $K_{1}, K_{2} \in P_{c p}(X)$ and $k \in \mathbb{N}^{*}$ arbitrarily chosen, but fixed. For $u_{1} \in K_{1}, u_{2} \in K_{2}$ and $\omega \in \Lambda_{k+m}(I)$, considering $u \in \Lambda_{m}(I)$ and $v \in \Lambda_{k}(I)$ with the property that $\omega=u v$, we get

$$
d\left(f_{\omega}\left(u_{1}\right), f_{\omega}\left(u_{2}\right)\right)=d\left(f_{u v}\left(u_{1}\right), f_{u v}\left(u_{2}\right)\right)=
$$




$$
\begin{gathered}
=d\left(f_{u}\left(f_{v}\left(u_{1}\right)\right), f_{u}\left(f_{v}\left(u_{2}\right)\right)\right) \stackrel{\beta) \text { from Def. } 3.1}{\leq} \\
\leq \sum_{w \in V_{m}} a_{u, w} d\left(f_{w}\left(f_{v}\left(u_{1}\right)\right), f_{w}\left(f_{v}\left(u_{2}\right)\right)\right)= \\
=\sum_{w \in V_{m}} a_{u, w} d\left(f_{w v}\left(u_{1}\right), f_{w v}\left(u_{2}\right)\right) \stackrel{\text { Defin. of } x_{n}}{\leq} \\
\leq a_{u} x_{k}+\sum_{w \in \Lambda_{1}(I)} a_{u, w} x_{k+1}+\ldots+\sum_{w \in \Lambda_{m-1}(I)} a_{u, w} x_{k+m-1} \leq \\
\leq \max \left\{x_{k+m-1}, \ldots, x_{k+1}, x_{k}\right\}\left(a_{u}+\sum_{w \in \Lambda_{1}(I)} a_{u, w}+\ldots+\sum_{w \in \Lambda_{m-1}(I)} a_{u, w}\right) \leq \\
\leq y_{k+m-1} \sum_{w \in V_{m}(I)} a_{u, w}=A_{u} y_{k+m-1} \leq A y_{k+m-1} .
\end{gathered}
$$

Consequently we obtain

$$
\max _{u_{1} \in K_{1}, u_{2} \in K_{2}} d\left(f_{\omega}\left(u_{1}\right), f_{\omega}\left(u_{2}\right)\right)=\delta\left(f_{\omega}\left(K_{1}\right), f_{\omega}\left(K_{2}\right)\right) \leq A y_{k+m-1},
$$

for every $\omega \in \Lambda_{k+m}(I)$ and therefore we get

$$
x_{k+m}=\sup _{\omega \in \Lambda_{k+m}(I)} \delta\left(f_{\omega}\left(K_{1}\right), f_{\omega}\left(K_{2}\right)\right) \leq A y_{k+m-1}<y_{k+m-1} .
$$

Taking into account that

$x_{k+1}, x_{k+2}, \ldots, x_{k+m-1} \leq \max \left\{x_{k+m-1}, \ldots, x_{k+1}, x_{k}\right\}=y_{k+m-1}$,

we conclude that $y_{k+m}=\max \left\{x_{k+m}, x_{k+m-1}, \ldots, x_{k+1}\right\} \leq y_{k+m-1}$.

THE SECOND STEP is to prove that the series $\sum_{k=m}^{\infty} s^{k} y_{k}\left(K_{1}, K_{2}\right)$ is convergent for all $K_{1}, K_{2} \in P_{c p}(X)$.

Justification of the second step. For $K_{1}, K_{2} \in P_{c p}(X)$ arbitrarily chosen, but fixed, we have

$$
\begin{gathered}
y_{k+2 m-1}=\max \left\{x_{k+2 m-1}, x_{k+2 m-2}, \ldots, x_{k+m}\right\} \stackrel{(1)}{\leq} \\
\leq \max \left\{A y_{k+2 m-2}, A y_{k+2 m-3}, \ldots, A y_{k+m-1}\right\} \stackrel{\text { first step }}{=} A y_{k+m-1},
\end{gathered}
$$

for every $k \in \mathbb{N}^{*}$. Hence $y_{j m+l} \leq A^{j-1} y_{m+l} \stackrel{\text { first step }}{\leq} A^{j-1} y_{m}$ for every $j \in \mathbb{N}^{*}$ and every $l \in\{0,1, \ldots, m-1\}$. The multiplication of the last inequality by $s^{j m+l}$ yields $s^{j m+l} y_{j m+l} \leq A^{j-1}\left(s^{m}\right)^{j-1} s^{l+m} y_{m} \leq\left(A s^{m}\right)^{j-1} s^{2 m} y_{m}$, i.e.

$$
s^{j m+l} y_{j m+l} \leq C^{j-1} s^{2 m} y_{m},
$$


for every $j \in \mathbb{N}^{*}$ and every $l \in\{0,1, \ldots, m-1\}$.

Let us note that for every $n \in \mathbb{N}, n \geq m$, there exists $j \in \mathbb{N}^{*}$ and $l \in\{0,1, \ldots, m-1\}$ such that $n=j m+l$, so

$$
\begin{gathered}
\sum_{k=m}^{n} s^{k} y_{k}=\sum_{k=m}^{2 m-1} s^{k} y_{k}+\sum_{k=2 m}^{3 m-1} s^{k} y_{k}+\ldots+\sum_{k=(j-1) m}^{j m-1} s^{k} y_{k}+\sum_{k=j m}^{n} s^{k} y_{k} \stackrel{(2)}{\leq} \\
\leq \sum_{k=m}^{2 m-1} s^{2 m} y_{m} C^{0}+\sum_{k=2 m}^{3 m-1} s^{2 m} y_{m} C^{1}+\ldots+\sum_{k=(j-1) m}^{j m-1} s^{2 m} y_{m} C^{j-2}+\sum_{k=j m}^{n} s^{2 m} y_{m} C^{j-1} \leq \\
\leq m s^{2 m} y_{m}\left(1+C+\ldots+C^{j-2}\right)+s^{2 m} y_{m}(n+1-j m) C^{j-1} \leq \\
\leq m s^{2 m} y_{m}\left(1+C+\ldots+C^{j-2}\right)+m s^{2 m} y_{m} C^{j-1}=m s^{2 m} y_{m}\left(1+C+\ldots+C^{j-1}\right)= \\
=m s^{2 m} y_{m} \frac{1-C^{j}}{1-C}<\frac{m s^{2 m} y_{m}}{1-C} .
\end{gathered}
$$

Consequently, the sequence of the partial sums of the series $\sum_{k=m}^{\infty} s^{k} y_{k}$ is bounded. As it is obviously increasing, we conclude that it is convergent, i.e. the series $\sum_{k=m}^{\infty} s^{k} y_{k}$ is convergent.

Let us note that, taking into account the comparison test, using (1), we infer that the series $\sum_{k=m+1}^{\infty} s^{k} x_{k}, \sum_{k=m+1}^{\infty} x_{k}$ and $\sum_{k=m+1}^{\infty} y_{k}$ are convergent. In particular, we deduce that $\lim _{k \rightarrow \infty} y_{k}\left(K_{1}, K_{2}\right)=\lim _{k \rightarrow \infty} x_{k}\left(K_{1}, K_{2}\right)=0$ for all $K_{1}, K_{2} \in P_{c p}(X)$.

THE THIRD STEP is to justify that the sequence $\left(F_{\mathcal{S}}^{[k]}(K)\right)_{k \in \mathbb{N}^{*}}$ is convergent for every $K \in P_{c p}(X)$.

Justification of the third step. Note for $K_{1}, K_{2} \in P_{c p}(X)$ arbitrarily chosen, we have

$$
\begin{gathered}
H\left(F_{\mathcal{S}}^{[k]}\left(K_{1}\right), F_{\mathcal{S}}^{[k]}\left(K_{2}\right)\right)=H\left(\bigcup_{\omega \in \Lambda_{k}(I)} f_{\omega}\left(K_{1}\right), \bigcup_{\omega \in \Lambda_{k}(I)} f_{\omega}\left(K_{2}\right)\right) \stackrel{\text { Prop. 2.4, ii })}{\leq} \\
\leq \max \left\{H\left(f_{\omega}\left(K_{1}\right), f_{\omega}\left(K_{2}\right)\right) \mid \omega \in \Lambda_{k}(I)\right\} \stackrel{\text { Remark 2.4 }}{\leq} x_{k} .
\end{gathered}
$$

Therefore, as $\lim _{k \rightarrow \infty} x_{k}\left(K_{1}, K_{2}\right)=0$, we get

$$
\lim _{k \rightarrow \infty} H\left(F_{\mathcal{S}}^{[k]}\left(K_{1}\right), F_{\mathcal{S}}^{[k]}\left(K_{2}\right)\right)=0 .
$$


Now, taking $K_{1}=K \in P_{c p}(X)$ and $K_{2}=F_{\mathcal{S}}(K) \in P_{c p}(X)$, based on (3), the convergence of the series $\sum_{k \in \mathbb{N}^{*}} s^{k} x_{k}$ and the comparison test, we come to the conclusion that the series $\sum_{k \in \mathbb{N}^{*}} s^{k} H\left(F_{\mathcal{S}}^{[k+1]}(K), F_{\mathcal{S}}^{[k]}(K)\right)$ is convergent for all $K \in P_{c p}(X)$. Hence, in view of Lemma 2.2, $\left(F_{\mathcal{S}}^{[k]}(K)\right)_{k \in \mathbb{N}^{*}}$ is a Cauchy sequence and, because $\left(P_{c p}(X), H, s\right)$ is complete (see Proposition 2.5), it is convergent.

The FOURTh STEP is to prove that all the sequences $\left(F_{\mathcal{S}}^{[k]}(K)\right)_{k \in \mathbb{N}^{*}}$, where $K \in P_{c p}(X)$, have the same limit.

Justification of the fourth step.

According to the third step, for every $K_{1}, K_{2} \in P_{c p}(X)$, there exist $A_{K_{1}}, A_{K_{2}} \in$ $\in P_{c p}(X)$ such that

$$
\lim _{k \rightarrow \infty} H\left(F_{\mathcal{S}}^{[k]}\left(K_{1}\right), A_{K_{1}}\right)=0 \text { and } \lim _{k \rightarrow \infty} H\left(F_{\mathcal{S}}^{[k]}\left(K_{2}\right), A_{K_{2}}\right)=0 .
$$

Since

$$
\begin{aligned}
& H\left(A_{K_{1}}, A_{K_{2}}\right) \stackrel{\text { Prop. } 2.5}{\leq} s\left(H\left(A_{K_{1}}, F_{\mathcal{S}}^{[k]}\left(K_{1}\right)\right)+H\left(F_{\mathcal{S}}^{[k]}\left(K_{1}\right), A_{K_{2}}\right)\right) \stackrel{\text { Prop. } 2.5}{\leq} \\
\leq & s H\left(A_{K_{1}}, F_{\mathcal{S}}^{[k]}\left(K_{1}\right)\right)+s^{2}\left(H\left(F_{\mathcal{S}}^{[k]}\left(K_{1}\right), F_{\mathcal{S}}^{[k]}\left(K_{2}\right)\right)+H\left(F_{\mathcal{S}}^{[k]}\left(K_{2}\right), A_{K_{2}}\right)\right),
\end{aligned}
$$

for all $k \in \mathbb{N}^{*}$, by passing to limit as $k$ goes to $\infty$, using (4) and (5) we get that $H\left(A_{K_{1}}, A_{K_{2}}\right)=0$, so $A_{K_{1}}=A_{K_{2}}$ for every $K_{1}, K_{2} \in P_{c p}(X)$.

Finally, denoting by $A_{\mathcal{S}}$ the common limit of the sequences $\left(F_{\S}^{[k]}(K)\right)_{k \in \mathbb{N}^{*}}$, where $K \in P_{c p}(X)$, we conclude that $\lim _{k \rightarrow \infty} H\left(F_{\mathcal{S}}^{[k]}(K), A_{\mathcal{S}}\right)=0$ for every $K \in P_{c p}(X)$

Proposition 3.2. For every iterated function system consisting of generalized convex contractions on a complete b-metric space $\mathcal{S}=\left((X, d, s), m,\left(f_{i}\right)_{i \in I}\right)$ and every $\omega \in \Lambda(I)$ there exists $A_{\omega} \in P_{c p}(X)$ such that

$$
\lim _{k \rightarrow \infty} H\left(f_{[\omega]_{k}}(K), A_{\omega}\right)=0,
$$

for every $K \in P_{c p}(X)$.

Proof. For $\omega \in \Lambda(I)$ and $K_{1}, K_{2} \in P_{c p}(X)$ arbitrarily chosen, but fixed, we have

$$
0 \leq H\left(f_{[\omega]_{n}}\left(K_{1}\right), f_{[\omega]_{n}}\left(K_{2}\right)\right) \stackrel{\text { Remark } 2.4}{\leq} \delta\left(f_{[\omega]_{n}}\left(K_{1}\right), f_{[\omega]_{n}}\left(K_{2}\right)\right) \leq x_{n}
$$


for every $n \in \mathbb{N}^{*}$.

The fact that $\lim _{n \rightarrow \infty} x_{n}=0$ yields

$$
\lim _{n \rightarrow \infty} \delta\left(f_{[\omega]_{n}}\left(K_{1}\right), f_{[\omega]_{n}}\left(K_{2}\right)\right)=\lim _{n \rightarrow \infty} H\left(f_{[\omega]_{n}}\left(K_{1}\right), f_{[\omega]_{n}}\left(K_{2}\right)\right)=0 .
$$

Since, for every $K_{1} \in P_{c p}(X)$, the series $\sum_{n \in \mathbb{N}^{*}} s^{n} x_{n}\left(K_{1}, F_{\delta}\left(K_{1}\right)\right)$ is convergent and

$$
\begin{gathered}
s^{n} H\left(f_{[\omega]_{n}}\left(K_{1}\right), f_{[\omega]_{n+1}}\left(K_{1}\right)\right) \stackrel{\text { Remark } 2.4}{\leq} s^{n} \delta\left(f_{[\omega]_{n}}\left(K_{1}\right), f_{[\omega]_{n+1}}\left(K_{1}\right)\right) \leq \\
\leq s^{n} \delta\left(f_{[\omega]_{n}}\left(K_{1}\right), f_{[\omega]_{n}}\left(F_{\mathcal{S}}\left(K_{1}\right)\right)\right) \leq s^{n} x_{n}\left(K_{1}, F_{\mathcal{S}}\left(K_{1}\right)\right)
\end{gathered}
$$

for each $n \in \mathbb{N}^{*}$, the comparison criterion assures us that the series $\sum_{n \in \mathbb{N}^{*}} s^{n} H\left(f_{[\omega]_{n}}\left(K_{1}\right), f_{[\omega]_{n+1}}\left(K_{1}\right)\right)$ is convergent. Based on Lemma 2.2, we infer that the sequence $\left(f_{[\omega]_{n}}\left(K_{1}\right)\right)_{n \in \mathbb{N}^{*}}$ is Cauchy and as, $\left(P_{c p}(X), H, s\right)$ is complete (see Proposition 2.5), there exists $A_{\omega}^{K_{1}} \in P_{c p}(X)$ such that

$$
\lim _{n \rightarrow \infty} H\left(f_{[\omega]_{n}}\left(K_{1}\right), A_{\omega}^{K_{1}}\right)=0 .
$$

Similarly, for $K_{2} \in P_{c p}(X)$, there exists $A_{\omega}^{K_{2}} \in P_{c p}(X)$ such that

$$
\lim _{n \rightarrow \infty} H\left(f_{[\omega]_{n}}\left(K_{2}\right), A_{\omega}^{K_{2}}\right)=0 .
$$

From (1), (2) and (3) we obtain that

$$
A_{\omega}^{K_{1}}=A_{\omega}^{K_{2}} \stackrel{\text { def }}{=} A_{\omega}
$$

for each $K_{1}, K_{2} \in P_{c p}(X)$.

Finally, by denoting by $A_{\omega}$ the common limit of the sequences $\left(F_{\mathcal{S}}^{[k]}(K)\right)_{k \in \mathbb{N}^{*}}$, where $K \in P_{c p}(X)$, we conclude that $\lim _{n \rightarrow \infty} H\left(f_{[\omega]_{n}}(K), A_{\omega}\right)=0$, for every $K \in P_{c p}(X)$.

The following two lemmas give more details about the convergence presented by Proposition 3.2.

Lemma 3.3. In the framework of Proposition 3.2, we have

$$
\lim _{n \rightarrow \infty} \sup _{\omega \in \Lambda(I)} H\left(f_{[\omega]_{n}}(K), A_{\omega}\right)=0,
$$

for every $K \in P_{c p}(X)$, i.e. the convergence described in the aforementioned Proposition is uniform with respect to $\omega \in \Lambda(I)$. 
Proof. Let us fix $K \in P_{c p}(X)$. We have

$$
\begin{gathered}
H\left(f_{[\omega]_{n}}(K), A_{\omega}\right) \stackrel{\text { Lemma } 2.1}{\leq} \\
\leq \sum_{k=n}^{p} s^{k-n+2} H\left(f_{[\omega]_{k}}(K), f_{[\omega]_{k+1}}(K)\right)+s H\left(f_{[\omega]_{p+1}}(K), A_{\omega}\right),
\end{gathered}
$$

for every $p, n \in \mathbb{N}, p \geq n$ and $\omega \in \Lambda(I)$.

Passing to limit as $p \rightarrow \infty$ in (1) and using Proposition 3.2, we obtain

$$
\begin{aligned}
& H\left(f_{[\omega]_{n}}(K), A_{\omega}\right) \leq \sum_{k \geq n} s^{k-n+2} H\left(f_{[\omega]_{k}}(K), f_{[\omega]_{k+1}}(K)\right) \stackrel{\text { Remark 2.14 }}{\leq} \\
& \leq \sum_{k \geq n} s^{k-n+2} \delta\left(f_{[\omega]_{k}}(K), f_{[\omega]_{k}}\left(F_{\mathcal{S}}(K)\right)\right) \leq \sum_{k \geq n} s^{k-n+2} x_{k}\left(K, F_{\mathcal{S}}(K)\right)
\end{aligned}
$$

for every $\omega \in \Lambda(I)$ and every $n \in \mathbb{N}$. Thus

$$
\sup _{\omega \in \Lambda(I)} H\left(f_{[\omega]_{n}}(K), A_{\omega}\right) \leq \sum_{k \geq n} s^{k-n+2} x_{k}\left(K, F_{\mathcal{S}}(K)\right)=s^{2-n} \sum_{k \geq 2} s^{k} x_{k}\left(K, F_{\mathcal{S}}(K)\right)
$$

for every $n \in \mathbb{N}$.

Taking into account the convergence of the series $\sum_{k} s^{k} x_{k}\left(K, F_{\mathcal{S}}(K)\right)$ and the fact that $\lim _{n \rightarrow \infty} s^{2-n}=0$, by passing to limit as $n \rightarrow \infty$ in (2) we conclude that $\lim _{n \rightarrow \infty} \sup _{\omega \in \Lambda(I)} H\left(f_{[\omega]_{n}}(K), A_{\omega}\right)=0$.

Lemma 3.4. In the framework of Proposition 3.2, the set $A_{\omega}$ has just one element which we denote by $a_{\omega}$.

Proof. Let us fix $K_{0} \in P_{c p}(X)$ and $\varepsilon>0$. Taking into account (1) from the proof of Proposition 3.2, we infer that $\lim _{n \rightarrow \infty} \operatorname{diam}\left(f_{[\omega]_{n}}\left(K_{0}\right)\right)=0$, so there exists $n_{\varepsilon}^{1} \in \mathbb{N}^{*}$ such that

$$
\operatorname{diam}\left(f_{[\omega]_{n}}\left(K_{0}\right)\right)<\frac{\varepsilon}{3},
$$

for all $n \in \mathbb{N}, n \geq n_{\varepsilon}^{1}$.

Proposition 3.2 provides $n_{\varepsilon}^{2} \in \mathbb{N}^{*}$ such that

$$
H\left(f_{[\omega]_{n}}\left(K_{0}\right), A_{\omega}\right)<\frac{\varepsilon}{3},
$$

for all $n \in \mathbb{N}, n \geq n_{\varepsilon}^{2}$. 
With the notation $n_{\varepsilon}=\max \left\{n_{\varepsilon}^{1}, n_{\varepsilon}^{2}\right\}$, from (2) and the definition of $H$, we get the existence of $\delta_{0}$ between 0 and $\frac{\varepsilon}{3}$ such that

$$
A_{\omega} \subseteq E_{\delta_{0}}\left(f_{[\omega]_{n_{\varepsilon}}}\left(K_{0}\right)\right)=\bigcup_{x \in f_{[\omega] n_{\varepsilon}}\left(K_{0}\right)} B\left(x, \delta_{0}\right) .
$$

For every $x, y \in A_{\omega}$, based on (3), there exist $u, v \in f_{[\omega]_{n_{\varepsilon}}}\left(K_{0}\right)$ such that $d(x, u)<\delta_{0}$ and $d(y, v)<\delta_{0}$. Hence

$$
\begin{gathered}
d(x, y) \leq s^{2}(d(x, u)+d(u, v)+d(v, y)) \leq \\
\leq s^{2}\left(2 \delta_{0}+\operatorname{diam}\left(f_{[\omega]_{n_{\varepsilon}}}\left(K_{0}\right)\right)\right) \stackrel{(1)}{<} s^{2}\left(2 \frac{\varepsilon}{3}+\frac{\varepsilon}{3}\right)=s^{2} \varepsilon .
\end{gathered}
$$

Thus

$$
\operatorname{diam}\left(A_{\omega}\right)=\sup _{x, y \in A_{\omega}} d(x, y) \leq s^{2} \varepsilon
$$

for every $\varepsilon>0$ and this implies that the set $A_{\omega}$ is a singleton.

The above Remarks can be synthesized in the following way:

$$
\lim _{n \rightarrow \infty} \sup _{\omega \in \Lambda(I)} H\left(f_{[\omega]_{n}}(K),\left\{a_{\omega}\right\}\right)=0
$$

for every $K \in P_{c p}(X)$.

The next proposition gives, for the case of strong $b$-metric spaces, a description of $A_{\mathcal{S}}$ using the elements $a_{\omega}$.

Proposition 3.5. For every iterated function system consisting of generalized convex contractions on a complete strong b-metric space $\mathcal{S}=$ $\left((X, d, s), m,\left(f_{i}\right)_{i \in I}\right)$, with the notations used in the above Remarks, $A_{\mathcal{S}}$ is the closure of the set $\left\{a_{\omega} \mid \omega \in \Lambda(I)\right\}$.

Proof. We are going to prove that $H\left(A_{\mathcal{S}}, \overline{\left\{a_{\omega} \mid \omega \in \Lambda(I)\right\}}\right)=0$, which, taking into account Proposition 2.5, implies the conclusion, i.e.

$$
A_{\mathcal{S}}=\overline{\left\{a_{\omega} \mid \omega \in \Lambda(I)\right\}} .
$$

Since

$$
\begin{gathered}
H\left(A_{\mathcal{S}},\left\{a_{\omega} \mid \omega \in \Lambda(I)\right\}\right) \stackrel{\text { Prop. 2.5 }}{\leq} \\
\leq s H\left(A_{\mathcal{S}}, F_{\mathcal{S}}^{[n]}(K)\right)+s H\left(F_{\mathcal{S}}^{[n]}(K),\left\{a_{\omega} \mid \omega \in \Lambda(I)\right\}\right) \leq s H\left(A_{\mathcal{S}}, F_{\mathcal{S}}^{[n]}(K)\right)+ \\
+s H\left(\bigcup_{\omega \in \Lambda_{n}(I)} \bigcup_{\alpha \in \Lambda(I)} f_{[\omega \alpha]_{n}}(K), \bigcup_{\omega \in \Lambda_{n}(I)} \bigcup_{\alpha \in \Lambda(I)}\left\{a_{\omega \alpha} \mid \alpha \in \Lambda(I)\right\}\right) \leq
\end{gathered}
$$




$$
\stackrel{\text { Prop.2.4, ii) }}{\leq} s H\left(A_{\mathcal{S}}, F_{\mathcal{S}}^{[n]}(K)\right)+s \max _{\omega \in \Lambda_{n}(I)} \sup _{\alpha \in \Lambda(I)} H\left(f_{\omega}(K),\left\{a_{\omega \alpha}\right\}\right)
$$

for all $n \in \mathbb{N}^{*}$ and $K \in P_{c p}(X), \lim _{n \rightarrow \infty} H\left(F_{\delta}^{[n]}(K), A_{\mathcal{S}}\right) \stackrel{\text { Prop. }}{=}{ }^{3.1} 0$ and

$\lim _{n \rightarrow \infty} \sup _{\omega \in \Lambda_{n}(I) \alpha \in \Lambda(I)} \sup _{\alpha} H\left(f_{\omega}(K),\left\{a_{\omega \alpha}\right\}\right) \stackrel{\text { Lemma }}{=}{ }^{3.3} 0$, by passing to limit as $n$ goes to $\infty$ in the above inequality, we infer that $H\left(A_{\delta},\left\{a_{\omega} \mid \omega \in \Lambda(I)\right\}\right)=0$ which implies, using Proposition 2.4, i), that $H\left(A_{\mathcal{S}}, \overline{\left\{a_{\omega} \mid \omega \in \Lambda(I)\right\}}\right)=0$.

Proposition 3.6. For every iterated function system consisting of generalized convex contractions on a complete strong $b$-metric space $\mathcal{S}=$ $\left((X, d, s), m,\left(f_{i}\right)_{i \in I}\right), F_{\S}$ is continuous.

Proof. We are going to prove that for every $\left(K_{n}\right)_{n \in \mathbb{N}} \subseteq P_{c p}(X)$ and $K \in$ $P_{c p}(X)$ the following implication is valid:

$$
\lim _{n \rightarrow \infty} H\left(K_{n}, K\right)=0 \Rightarrow \lim _{n \rightarrow \infty} H\left(F_{\mathcal{\delta}}\left(K_{n}\right), F_{\mathcal{S}}(K)\right)=0 .
$$

Indeed, let us fix an arbitrary $\varepsilon>0$.

Let us note that the continuity of the functions $f_{i}$ on $L \stackrel{\text { def }}{=} K \cup\left(\bigcup_{n=0}^{\infty} K_{n}\right)$ $\stackrel{\text { Prop. }}{\in}{ }^{2.6} P_{c p}(X)$, assures the existence of $\delta_{\varepsilon}>0$ such that

$$
d\left(f_{i}(x), f_{i}(y)\right)<\varepsilon
$$

for every $i \in I$ and every $x, y \in L$ such that $d(x, y)<\delta_{\varepsilon}$.

Since $\lim _{n \rightarrow \infty} H\left(K_{n}, K\right)=0$, there exists $n_{\varepsilon} \in \mathbb{N}$ such that

$$
H\left(K_{n}, K\right)<\frac{\delta_{\varepsilon}}{2}
$$

for every $n \in \mathbb{N}, n \geq n_{\varepsilon}$.

Let us consider $i \in I$ and $n \in \mathbb{N}, n \geq n_{\varepsilon}$.

For every $x \in K_{n}$ there exists $y_{x} \in K$ such that

$$
d\left(x, y_{x}\right)<\inf _{y \in K} d(x, y)+\frac{\delta_{\varepsilon}}{2}<H\left(K_{n}, K\right)+\frac{\delta_{\varepsilon}}{2} \stackrel{(2)}{<} \frac{\delta_{\varepsilon}}{2}+\frac{\delta_{\varepsilon}}{2}=\delta_{\varepsilon},
$$

so, taking into account (1), we have $\inf _{v \in f_{i}(K)} d\left(f_{i}(x), v\right) \leq d\left(f_{i}(x), f_{i}\left(y_{x}\right)\right)<\varepsilon$. Consequently $\sup _{x \in K_{n}}\left(\inf _{v \in f_{i}(K)} d\left(f_{i}(x), v\right)\right) \leq \varepsilon$, i.e.

$$
\sup _{u \in f_{i}\left(K_{n}\right)}\left(\inf _{v \in f_{i}(K)} d(u, v)\right) \leq \varepsilon .
$$


Similarly we obtain

$$
\sup _{u \in f_{i}(K)}\left(\inf _{v \in f_{i}\left(K_{n}\right)} d(u, v)\right) \leq \varepsilon
$$

Thus

$$
\begin{gathered}
H\left(F_{\mathcal{S}}\left(K_{n}\right), F_{\mathcal{S}}(K)\right) \stackrel{\text { Prop. 2.4, ii) }}{\leq} \max _{i \in I} H\left(f_{i}\left(K_{n}\right), f_{i}(K)\right)= \\
=\max _{i \in I}\left\{\max \left\{\sup _{u \in f_{i}\left(K_{n}\right)}\left(\inf _{v \in f_{i}(K)} d(u, v)\right), \sup _{u \in f_{i}(K)}\left(\inf _{v \in f_{i}\left(K_{n}\right)} d(u, v)\right)\right\}\right\} \stackrel{(3) \stackrel{\&}{\leq}(4)}{\leq} \varepsilon,
\end{gathered}
$$

for every $n \in \mathbb{N}, n \geq n_{\varepsilon}$. In other words, $\lim _{n \rightarrow \infty} H\left(F_{\mathcal{S}}\left(K_{n}\right), F_{\mathcal{S}}(K)\right)=0$.

Proposition 3.7. For every iterated function system consisting of generalized convex contractions on a complete strong b-metric space $\mathcal{S}=\left((X, d, s), m,\left(f_{i}\right)_{i \in I}\right)$, $F_{\mathcal{S}}$ is a Picard operator.

Proof. Let us fix $K \in P_{c p}(X)$.

Since, according to Proposition 3.1, we have $\lim _{k \rightarrow \infty} H\left(F_{\mathcal{S}}^{[k]}(K), A_{\mathcal{S}}\right)=0$, using Proposition 3.6, we deduce that $\lim _{k \rightarrow \infty} H\left(F_{\mathcal{S}}\left(F_{\mathcal{S}}^{[k]}(K)\right), F_{\mathcal{S}}\left(A_{\mathcal{S}}\right)\right)=0$, i.e. $\lim _{k \rightarrow \infty} H\left(F_{\mathcal{S}}^{[k+1]}(K), F_{\mathcal{S}}\left(A_{\mathcal{S}}\right)\right)=0$.

Uniqueness of the limit of a sequence assures us that $A_{\mathcal{S}}$ is a fixed point of $F_{\delta}$.

If $A_{1} \in P_{c p}(X)$ is a fixed point of $F_{\mathcal{S}}$, we have $F_{\delta}^{[k]}\left(A_{1}\right)=A_{1}$, so

$\lim _{k \rightarrow \infty} H\left(F_{\mathcal{S}}^{[k]}\left(A_{1}\right), A_{1}\right)=H\left(A_{1}, A_{1}\right)=0$.

But $\lim _{k \rightarrow \infty} H\left(F_{\mathcal{S}}^{[k]}\left(A_{1}\right), A_{\S}\right) \stackrel{\text { Prop. }}{=}{ }^{3.1} 0$, and, again, the uniqueness of the limit of a sequence implies that $A_{1}=A_{\mathcal{S}}$, i.e $A_{\mathcal{S}}$ is the unique fixed point of $F_{\mathcal{S}}$.

We conclude that there is a unique $A_{\mathcal{S}} \in P_{c p}(X)$ such that $F_{\mathcal{S}}\left(A_{\mathcal{S}}\right)=A_{\mathcal{S}}$ and $\lim _{k \rightarrow \infty} H\left(F_{\delta}^{[k]}(K), A_{\delta}\right)=0$ for every $K \in P_{c p}(X)$, i.e. $F_{\delta}$ is a Picard operator.

\section{Final remarks}

We can reformulate the previous proposition in the following way:

Theorem 4.1. Every iterated function system consisting of generalized convex contractions on a complete strong b-metric space has a unique attractor. 
Remark 4.1. Proposition 3.1 explains why $A_{\mathcal{S}}$ is called the attractor of $\mathcal{S}$, namely because it "attracts" all the elements of $P_{c p}(X)$.

Remark 4.2. The general method to prove the existence and uniqueness of the attractor of an iterated function system $\mathcal{S}$ consisting on elements from a certain class of contractions which are Picard operators is to show that $F_{\mathcal{S}}$ belongs to that class. As a consequence, one can infer that $F_{\mathcal{S}}$ has a unique fixed point $A_{\mathcal{S}}$ and that

$$
\lim _{k \rightarrow \infty} H\left(F_{\delta}^{[k]}(K), A_{\delta}\right)=0,
$$

for every $K \in P_{c p}(X)$.

Our approach was totally different, namely we first proved $(*)$ (see Proposition 3.1) and then, using it, we come to the conclusion that $A_{\S}$ is the unique fixed point of $F_{\mathcal{S}}$.

Remark 4.3. The case $s=1$ was treated in [22]. In case that the set $I$ has one element, $\left((X, d, 1), m,\left(f_{i}\right)_{i \in I}\right)$ is nothing else but the notion of generalized convex contraction. The notion of iterated function system consisting of convex contractions from [39] is a particular case of the one of iterated function system consisting of generalized convex contractions (just take $m=2$ ).

Remark 4.4. Proposition 3.1, Proposition 3.2 and Lemma 3.4 are valid under certain weaker hypotheses. More precisely, the proofs of the aforementioned results are valid if instead of $s$ we use $a \in\left(1, \sqrt[m]{\frac{1}{A}}\right)$. Consequently, hypothesis $\alpha$ ) from Definition 3.1 can be replaced by the following one: $\max _{\omega \in \Lambda_{m}(I)} \sum_{v \in V_{m}(I)} a_{\omega, v}<1$.

\section{References}

[1] Aghajani A., Abbas M., and Roshan J.R., Common fixed point of generalized weak contractive mappings in partially ordered b-metric spaces, Math. Slovaca, 64, (2014), 941-960.

[2] Alghamdia M.A., Alnafeia S.H., Radenović S., and Shahzad N., Fixed point theorems for convex contraction mappings on cone metric spaces, Math.Comput. Modelling, 54, (2011), 2020-2026.

[3] An T.V., Tuyen L.Q., and Dung N.V., Stone-type theorem on $b$-metric spaces and applications, Topology Appl., 185/186, (2015), 50-64.

[4] András Sz., Fiber Picard operators and convex contractions, Fixed Point Theory, 4, (2003), 121-129. 
[5] András Sz., Ecuaţii integrale Fredholm-Volterra, Editura Didactică şi Pedagogică, Bucureşti, 2005.

[6] Andres J. and Rypka M., Multivalued fractals and hyperfractals, Internat. J. Bifur. Chaos Appl. Sci. Engrg., 22, (2012), DOI 10.1142/S02181127412500095.

[7] Aydi H., Bota M.F., Karapinar E., and Mitrović S., A fixed point theorem for set-valued quasi-contractions in b-metric spaces, Fixed Point Theory Appl., (2012), 2012-88.

[8] Bakhtin I.A., The contraction mapping principle in quasimetric spaces, Funct. Anal., Unianowsk Gos. Ped. Inst., 30, (1989), 26-37.

[9] Berinde V., Generalized contractions in quasimetric spaces, Seminar on Fixed Point Theory, (1993), 3-9.

[10] Boriceanu M., Bota M., and Petruşel A., Multivalued fractals in $b$-metric spaces, Cent. Eur. J. Math., 8, (2010), 367-377.

[11] Boriceanu M., Petruşel A., and Rus A.I., Fixed point theorems for some multivalued generalized contraction in b-metric spaces, Int. J. Math. Stat., 6, (2010), 65-76.

[12] Bota M., Molnár A., and Varga C., On Ekeland's variational principle in b-metric spaces, Fixed Point Theory, 12, (2011), 21-28.

[13] Chifu C. and Petruşel A., Multivalued fractals and generalized multivalued contractions, Chaos Solitons Fractals, 36, (2008), 203-210.

[14] Chifu C. and Petruşel G., Fixed points for multivalued contractions in $b$-metric spaces with applications to fractals, Taiwanese J. Math., 18, (2014), 1365-1375.

[15] Czerwik S., Contraction mappings in b-metric spaces, Acta Math. Inform. Univ. Ostraviensis, 1, (1993), 5-11.

[16] Czerwik S., Nonlinear set-valued contraction mappings in b-metric spaces, Atti Sem. Mat. Fis. Univ. Modena, 46, (1998), 263-276.

[17] Dubey A.K., Shukla R., and Dubey R.P., Some fixed point results in $b$-metric spaces, Asian Journal of Mathematics and Applications, (2014), Article ID ama0147

[18] Dumitru D., Generalized iterated function systems containing Meir-Keeler functions, An. Univ. Bucur., Mat., 58, (2009), 109-121.

[19] Istrăţescu V., Some fixed point theorems for convex contraction mappings and convex nonexpansive mappings (I), Libertas Math., 1, (1981), 151-164.

[20] V. Istrățescu, Some fixed point theorems for convex contraction mappings and mappings with convex diminishing diameters - I, Annali di Mat. Pura Appl., 130, (1982), 89-104.

[21] V. Istrățescu, Some fixed point theorems for convex contraction mappings and mappings with convex diminishing diameters, II, Annali di Mat. Pura Appl., 134, (1983), 327-362.

[22] Georgescu F., IFSs consisting of generalized convex contractions, An. Ştiinţ. Univ. "Ovidius" Constanţa, Ser. Mat., in print 
[23] Ghorbanian V., Rezapour S., and N. Shahzad, Some ordered fixed point results and the property ,(P), Comput. Math. Appl., 63, (2012), 1361-1368.

[24] Gwóźdź-Łukowska G. and J. Jachymski, IFS on a metric space with a graph structure and extensions of the Kelisky-Rivlin theorem, J. Math. Anal. Appl., 356, (2009), 453-463.

[25] N. Hussain, M.A. Kutbi, Khaleghizadeh S., and P. Salimi, Discussions on recent results for $\alpha-\Psi$-contractive mappings, Abstr. Appl.Anal., 2014, (2014), Article ID 456482, 13 pages.

[26] Hutchinson J.E., Fractals and self similarity, Indiana Univ. Math. J., 30, (1981), 713-747.

[27] Khamsi M.A. and N. Hussain, KKM mappings in metric type spaces, Nonlinear Anal., 73, (2010), 3123-3129.

[28] Kir M. and Kizitune H., On some well known fixed point theorems in $b$-metric spaces, Turkish Journal of Analysis and Number Theory, 1, (2013), 13-16.

[29] Kirk W.A. and N. Shahzad, Fixed Point Theory in Distance Spaces, Springer Heidelberg, 2014.

[30] Klimek M. and Kosek M., Generalized iterated function systems, multifunctions and Cantor sets, Ann. Polon. Math., 96, (2009), 25-41.

[31] Kutbi M.A., Karapinar E., Ahmad J., and Azam A., Some fixed point results for multi-valued mappings in b-metric spaces, J. Inequal. Appl, 2014, (2014), article ID 126.

[32] Latif A., Sintunavarat W., and Ninsri A., Approximate fixed point theorems for partial generalized convex contraction mappings in $\alpha$-complete metric spaces, Taiwanese J. Math., 19, (2015), 315-333.

[33] Leśniak K., Infinite iterated function systems: a multivalued approach, Bull. Pol. Acad. Sci. Math., 52, (2004), 1-8.

[34] Máté L., The Hutchinson-Barnsley theory for certain noncontraction mappings, $P e$ riod. Math. Hungar., 27, (1993), 21-33.

[35] Miandaragh M.A., Postolache M., and Rezapour S., Approximate fixed points of generalized convex contractions, Fixed Point Theory Appl., 2013, (2013), article 255.

[36] Mihail A. and Miculescu R., Applications of fixed point theorems in the theory of generalized IFS, Fixed Point Theory Appl., (2008), Art. ID 312876, 11 pp.

[37] Generalized IFSs on noncompact spaces A. Mihail and R. Miculescu, Fixed Point Theory Appl., (2010), Art. ID 584215, 15 pp.

[38] Miculescu R. and Mihail A., Reich-type iterated function systems, J. Fixed Point Theory Appl., 18, (2016), 285-296.

[39] Miculescu R. and Mihail A., A generalization of Istrăţescu's fixed point theorem for convex contractions, in print, Fixed Point Theory (available at arXiv:1512.05490).

[40] Miculescu R. and Mihail A., Caristi-Kirk type and Boyd\&Wong-BrowderMatkowski-Rus type fixed point results in b-metric spaces, in print, Filomat (available at arXiv:1512.03968) 
[41] Mishra P.K., Sachdeva S., and Banerjee S.K., Some fixed point theorems in b-metric space, Turkish Journal of Analysis and Number Theory, 2, (2014), 19-22.

[42] Mohanta S.K., Some fixed point theorems using wt-distance in $b$-metric spaces, Fasc. Math., 54, (2015), 125-140.

[43] Mureşan V. and Mureşan A., On the theory of fixed point theorems for convex contraction mappings, Carpathian J. Math., 31, (2015), 365-371.

[44] Nashine H.N. and Kadelburg Z., Cyclic generalized $\varphi$-contractions in b-metric spaces and an application to integral equations, Filomat, 28, (2014), 2047-2057.

[45] Nazir T., Silvestrov S., and Qi Xiaomin, Fractals of generalized $F$-Hutchinson operator in b-metric spaces, J. Oper., 2016, (2016), article ID 5250394.

[46] Olatinwo M.O., A fixed point theorem for multi-valued weakly Picard operators in b-metric spaces, Demonstratio Math., 42, (2009), 599-606.

[47] Păcurar M., Sequences of almost contractions and fixed points in $b$-metric spaces, An. Univ. Vest Timiş. Ser. Mat.-Inform., 48, (2010), 125-137.

[48] Petruşel A., Iterated function system of locally contractive operators, Rev. Anal. Numér. Théor. Approx., 33, (2004), 215-219.

[49] Roshan J.R., Hussain N., Sedghi S., and Shobkolaei N., Suzuki-type fixed point results in b-metric spaces, Math. Sci. (Springer), 9, (2015), 153-160.

[50] Roshan J.R., Parvaneh V., and Altun I., Some coincidence point results in ordered $b$-metric spaces and applications in a system of integral equations, Appl.Math.Comput., 226, (2014), 725-737.

[51] M. Sarwar and Rahman M.U., Fixed point theorems for Ciric's and generalized contractions in b-metric spaces, International Journal of Analysis and Applications, 7, (2015), 70-78.

[52] N.A.. Secelean, Iterated function systems consisting of F-contractions, Fixed Point Theory Appl., (2013), 2013-277.

[53] Secelean N.A., Generalized iterated function systems on the space $l^{\infty}(X)$, J. Math. Anal. Appl., 410, (2014), 847-458.

[54] Secelean N.A., Countable Iterated Function Systems, Lambert Academic Publishing, 2013

[55] Shukla S., Partial b-metric spaces and fixed point theorems, Mediterr. J. Math., 11, (2014), 703-711.

[56] Singh S.L., Czerwik S., Król K., and Singh A., Coincidences and fixed points of hybrid contractions, Tamsui Oxf. J. Math. Sci., 24, (2008), 401-416.

[57] Strobin F. and J. Swaczyna, On a certain generalization of the iterated function systems, Bull. Australian Math. Soc., 87, (2013), 37-54.

[58] Strobin F., Attractors of generalized IFSs that are not attractors of IFSs, J. Math. Anal. Appl., 422, (2015), 99-108.

[59] Yingtaweesittikul H., Suzuki type fixed point for generalized multi-valued mappings in b-metric spaces, Fixed Point Theory and Applications, (2013), 215. 


\section{Flavian Georgescu}

Faculty of Mathematics and Computer Science

University of Piteşti, Romania

Târgul din Vale 1, 110040, Piteşti, Argeş, Romania

E-mail: georgescu.flavian@ichb.ro

Received: 26.04.2017

Accepted: 26.10.2017 